Article type : Regular Paper

\title{
CARDIOPROTECTIVE ROLE OF IGF-1 IN THE HYPERTROPHIED MYOCARDIUM OF THE SPONTANEOUSLY HYPERTENSIVE RATS: A KEY EFFECT ON NHE-1 ACTIVITY
}

Alejandra M. Yeves, Juan I. Burgos, Andrés J. Medina, M. Celeste Villa-Abrille; Irene L. Ennis

Centro de Investigaciones Cardiovasculares. Facultad de Ciencias Médicas. UNLPCONICET. Argentina

Short title: IGF-1 effect on the SHR myocardial NHE-1.

\section{Address for correspondence:}

Irene Lucía Ennis, MD, PhD.

Centro de Investigaciones Cardiovasculares

Facultad de Ciencias Médicas, UNLP.

Calle 60 y 120, 1900, La Plata. Argentina

Fax/Phone: 54-221-483-4833

e-mail: iennis@med.unlp.edu.ar

This article has been accepted for publication and undergone full peer review but has not been through the copyediting, typesetting, pagination and proofreading process, which may lead to differences between this version and the Version of Record. Please cite this article as doi: 10.1111/apha.13092

This article is protected by copyright. All rights reserved. 


\section{ABSTRACT}

Aim: Myocardial $\mathrm{Na}^{+} / \mathrm{H}^{+}$exchanger-1 (NHE-1) hyperactivity and oxidative stress are interrelated phenomena playing pivotal roles in the development of pathological cardiac hypertrophy and heart failure. Exercise training is effective to convert pathological into physiological hypertrophy in the spontaneously hypertensive rats (SHR) and IGF-1 -key humoral mediator of exercise training- inhibits myocardial NHE-1, at least in normotensive rats. Therefore, we hypothesize that IGF-1 by hampering NHE-1 hyperactivity and oxidative stress should exert a cardioprotective effect in the SHR. Methods: NHE-1 activity [proton efflux $\left(\mathrm{J}_{\mathrm{H}}^{+}\right) \mathrm{mmol} L \mathrm{~min}-1$ ], expression and phosphorylation; $\mathrm{H}_{2} \mathrm{O}_{2}$ production; superoxide dismutase (SOD) activity; contractility and calcium transients were measured in SHR hearts in the presence/absence of IGF-1. Results: IGF-1 significantly decreased NHE-1 activity $\left(\mathrm{J}_{\mathrm{H}}{ }^{+}\right.$at $\mathrm{pH}_{\mathrm{i}}$ 6.95: $1.39 \pm 0.32, \mathrm{n}=9$ vs. $\left.\mathrm{C} 3.27 \pm 0.3, \mathrm{n}=20, \mathrm{p}<0.05\right)$; effect prevented by AG1024, an antagonist of IGF-1 receptor $(2.7 \pm 0.4, n=7)$; by the PI3K inhibitor wortmannin (3.14 $\pm 0.41 \mathrm{n}=7)$; and the AKT inhibitor MK2206 (3.37 $\pm 0.43, \mathrm{n}=14)$. Moreover, IGF-1 exerted an antioxidant effect revealed by a significant reduction in $\mathrm{H}_{2} \mathrm{O}_{2}$ production accompanied by an increase in SOD activity. In addition, IGF-1 improved cardiomyocyte contractility as evidenced by an increase in sarcomere shortening and a decrease in the relaxation constant, underlined by an increase in the amplitude and rate of decay of the calcium transients.

Conclusions: IGF-1 exerts a cardioprotective role on the hypertrophied hearts of the SHR, in which the inhibition of NHE-1 hyperactivity, as well as the positive inotropic and antioxidant effects, emerge as key players. 
Key words: contractility, hypertension; insulin like growth factor 1; NHE-1; oxidative stress.

\section{INTRODUCTION}

There is strong evidence supporting that the $\mathrm{Na}+/ \mathrm{H}+$ exchanger-1 $(\mathrm{NHE}-1)$ is critically involved in cardiac diseases, especially in pathological cardiac hypertrophy and its evolution to heart failure. ${ }^{1,2}$ The NHE-1 catalyzes the exchange of intracellular $\mathrm{H}^{+}$for extracellular $\mathrm{Na}^{+}$. Its functional up-regulation is sufficient to induce pathological cardiac hypertrophy while its specific inhibition represents an efficient approach to induce cardiac hypertrophy prevention or regression. ${ }^{2-4}$

The spontaneously hypertensive rats (SHR) are a well-known model of essential hypertension that results in pathological cardiac hypertrophy. We and others have demonstrated that the NHE-1 is hyperactive in the hypertrophied myocardium of these rats. ${ }^{5,} 6$ Myocardial stretch due to hemodynamic overload stimulates an autocrine/paracrine mechanism that involves the activation of the ERK/p90 ${ }^{\mathrm{RSK}}$ pathway conducting to NHE-1 phosphorylation and hyperactivity. 2,7 The resulting increase in intracellular $\mathrm{Na}^{+}$content decreases the forward and facilitates the reverse mode of operation of the $\mathrm{Na}^{+} / \mathrm{Ca}^{2+}$ exchanger, ultimately promoting the activation of calcineurin. The latter is a prohypertrophic phosphatase critical for pathological cardiac hypertrophy development. Interestingly, in the SHR we have demonstrated that sustained exercise training is capable of transforming the hypertension-induced pathological cardiac hypertrophy into a physiological one, improving not only myocardial structure but also cardiac function at the time that calcineurin activity is normalized. ${ }^{8}$ 
The physiologic adaptation to exercise involves the production and release of several growth factors and other humoral mediators among which, the insulin-like growth factor 1 (IGF-1) seems to be the most relevant. It has been reported that exercise training rises the levels of IGF-1 in athletes as well as in animal models. ${ }^{9-11}$ The importance of the IGF-1 in the development of physiological cardiac hypertrophy has been also confirmed by evidence arose from transgenic mice overexpressing the IGF1 receptor (IGF-1R) in the heart or, on the contrary, with cardiomyocyte-specific knockout of this receptor. ${ }^{12,13}$ The activation of this membrane receptor stimulates at least to main signalling cascades: the PI3K/AKT pathway, the most important in exercise-induced physiological hypertrophy development, and the ERK pathway. ${ }^{14}$ Accordingly, physiological cardiac hypertrophy ${ }^{12}, 15,16$ can be eluded by pharmacological or genetic inhibition of PI3K or AKT1, while the opposite occurs when a constitutively active PI3K is overexpressed in the heart. ${ }^{12,15,16} 17$ With respect to the contractile adaptation, we recently reported in mice cardiomyocytes that IGF-1 induces a positive inotropic and lusitropic effect that was mediated by the activation of the calcium-calmodulin kinase II (CaMKII) and the phosphorylation of phospholamban (PLN). ${ }^{18}$

Interestingly, we have recently reported that IGF-1, through an AKT-dependent mechanism, exerts an inhibitory action upon the NHE-1 in cardiomyocytes from normotensive rats (Wistar). ${ }^{19}$ Moreover, in cardiomyocytes from these rats after being subjected to a swimming routine, and therefore hemodynamic overload, we did not detect calcineurin/NFAT activation, suggesting that NHE-1 hyperactivity, characteristic of pathological hypertrophy, was prevented in this model of cardiac hypertrophy. ${ }^{19}$ 
Large amount of evidence support the idea that reactive oxygen species (ROS) play a key role in the development of pathological cardiac hypertrophy and its progression to failure. ${ }^{20,21}$ However, the underlying molecular mechanism involved remains to be elucidated. Mitochondria are the main source of ROS production, while the NADPH oxidase, xanthine oxidase and the uncoupled nitric oxide synthase are the main extra mitochondrial sources. The SHR myocardium is characterized by augmented oxidative damage compared to the myocardium of normotensive rats, evidenced by an increase in lipid peroxidation (TBARS), protein nitration and NADPH oxidase activity. $^{22}$ Interestingly, we have previously demonstrated that pharmacologic inhibition of the NHE-1 was able to decrease myocardial ROS production in cat myocardium by a direct mitochondrial action. ${ }^{23}$

Based on this evidence we hypothesise that IGF-1, through impeding NHE-1 hyperactivity and improving redox balance, would be a key player in the cardioprotective effect of training upon the hypertrophied myocardium of the SHR. A main component of this effect would be an AKT-dependent inhibitory phosphorylation of the NHE-1.

\section{RESULTS}

The activity of the NHE-1 was explored in isolated cardiomyocytes from SHR and normotensive Wistar rats. As expected, the recovery from an induced intracellular acidosis was significantly faster in SHR than in Wistar cardiomyocytes, confirming that this ion exchanger is hyperactive in the hypertrophied myocardium of the SHR (Figure 1A). Since the exchanger hyperactivity could be the result of increased NHE-1 expression and/or a post-translational modification of the protein, both possibilities were evaluated. We found that the NHE-1 was similarly expressed in the hypertrophied myocardium of the SHR and the normotrophic one of the Wistar rats 
(Figure 1B); however, we detected a significant increase in the phosphorylation state of the cytosolic tail residue Ser703, a modification widely recognized to stimulate NHE-1 activity (Figure 1C, left panel). ${ }^{24,25}$ Since it has also been described an inhibitory AKT-dependent phosphorylation of the exchanger at the residue Ser648, ${ }^{19,26}$ we explore this site too. As it can be appreciated in Figure 1C, right panel no significant differences were observed at this point. In line with these data, a greater activation of the ERK1/2 pathway, responsible for Ser703 phosphorylation, was observed in the myocardium of SHR compared to Wistar rats. On the contrary, no difference was detected in the activation of AKT (Figure 1D).

Since our aim was to reveal in hypertrophied hearts a putative effect of IGF-1 on the NHE-1, we explore the exchanger activity in isolated SHR cardiomyocytes after 24 hs. of culture with/without IGF-1. In agreement with our previous report in nonhypertrophied cardiomyocytes, ${ }^{19}$ a significant reduction in NHE-1 dependent proton efflux was observed in the SHR cardiomyocytes exposed to IGF-1. In order to get insight into the molecular mechanism involved, NHE-1 activity was measured in the presence of different inhibitors. The effect of IGF-1 was avoided not only by AG1024, an antagonist of the IGF-1R, but also by preventing PI3K or AKT activation with wortmannin and MK2206, respectively (Figure 2A-B). None of these inhibitors exert a significant effect on NHE-1 activity in the absence of IGF-1 $\left(\mathrm{J}_{\mathrm{H}}^{+}\right.$at $\mathrm{pH}_{\mathrm{i}} 6.95$ : $4.293 \pm 1.598 \mathrm{n}=5 ; 5.339 \pm 1.058 \mathrm{n}=9 ; 3.685 \pm 1.393 \mathrm{n}=7 ;$ and $2.991 \pm 0.574 \mathrm{n}=7$ for AG1024, wortmannin, MK2206 and DMSO respectively). On the other hand, the decrease in the activity of the NHE-1 induced by IGF-1 could not be attributed to a modification in either NHE-1 expression or the buffer capacity as shown in Figure 2C-D, respectively. Moreover, the values of $\mathrm{pH}_{\mathrm{i}}$ at the maximal acidification reached were not different among the experimental groups (control: 6.925 \pm 0.024 ; IGF-1: 


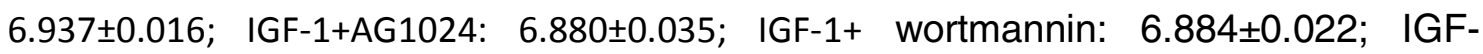
1+MK2206: 6.893 \pm 0.038$)$.

These functional data was supported by the results obtained when exploring the consequences of IGF-1 stimulation on IGF-1R, AKT and ERK1/2-p90RSK phosphorylation state. IGF-1 significantly increased the phosphorylation of all these signaling molecules (Figure 3A-D). Importantly, these results were in agreement with the phosphorylation measured at the cytosolic tail of the exchanger, where an increase in the AKT-dependent inhibitory site (Ser648) was detected with no difference at the stimulatory residue Ser703, target of the ERK1/2-p90RSK pathway (Figure 3E).

Previous results of our group demonstrated that chronic swimming has the ability to improve cardiac contractile function in the $\mathrm{SHR}^{8}$ therefore in a new set of experiments we explored the consequences of IGF-1 on contractility. By measuring sarcomere length in SHR isolated ventricular myocytes we were able to detect a positive inotropic effect of IGF-1 (Figure 4A-B). Interestingly, IGF-1 also seemed to improve cardiomyocyte relaxation as revealed by a decrease in the relaxation time constant, Tau (Figure 4C). These effects were prevented by the blockade of the IGF-1R with AG1024 and by preventing AKT activation with MK2206. In order to gain further insight into the mechanism underlying the positive inotropic/lusitropic effect of IGF-1, of IGF-1, calcium transients were recorded in isolated cardiomyocytes. As shown in Figure 5A-C and in agreement with previous findings of our group ${ }^{18}$, IGF-1 significantly increased the amplitude and rate of decay of the calcium transients. Based on these results, ${ }^{18}$ we next explored the involvement of CaMKII. To this aim, we measured the phosphorylation level of this kinase as well as of residue threonine 
17 (Thr17) of PLN, its well-recognized downstream target. We were able to detect a significant increase in in the phosphorylation of both proteins in the presence of IGF1 that was efficiently prevented by antagonizing the IGF-1R with $A G$. On the contrary, we did not detect a significant difference in the phosphorylation of the PKA site on PLN (Ser16) (Figure 5D).

Oxidative stress is another distinctive feature of pathological cardiac hypertrophy. Therefore, we investigated the effect of IGF-1 upon SHR myocardial $\mathrm{H}_{2} \mathrm{O}_{2}$ accumulation and the activity of the antioxidant enzymes SOD. IGF-1 significantly attenuated $\mathrm{H}_{2} \mathrm{O}_{2}$ fluorescence (Figure 6A-B) while it increased the activity of myocardial SOD by an AKT-dependent mechanism (Figure 6C-D). Neither AG1024 nor MK2206 significantly influenced SOD activity in the absence of IGF-1 $(27 \pm 2 n=4$ and $25 \pm 2 n=4$, respectively).

\section{DISCUSSION}

The main contribution of the present work is the evidence provided about the subcellular mechanisms underlying the beneficial effects of IGF-1 on the hypertrophied myocardium of the SHR. Herein, we demonstrated that IGF-1: 1prevents myocardial NHE-1 hyperactivity through an AKT-dependent inhibitory phosphorylation of the cytosolic tail of the exchanger; 2- improves sarcomere shortening and relaxation by increasing the amplitude and the rate of decay of calcium transients; and 3- improves redox state by decreasing $\mathrm{H}_{2} \mathrm{O}_{2}$ accumulation and augmenting SOD activity.

In 2009 , we reported that a 60 day period of swimming training was able to convert SHR pathological cardiac hypertrophy into a physiological phenotype, improving not only myocardial structure but also cardiac function. ${ }^{8}$ However, at that 
moment we did not explore the underling mechanism involved in the beneficial cardiac consequences of exercise. Based on our present results, it is tempting to speculate that the events schematized in Figure 7 may also explain the cardioprotection observed in trained $\mathrm{SHR}^{8}$ through the release of IGF-1.

IGF-1 is a 70 amino acid growth factor produced by most, if not all, body tissues including cardiomyocytes. Cardiac formation of IGF-1 -that is higher in athletes than in control subjects- seems to be the main player in physiological cardiac hypertrophy. ${ }^{10}$ Apart from its role in stimulating cardiomyocyte growth, we have recently demonstrated that IGF-1 can inhibit cardiac NHE-1 hyperactivity in normal hearts. ${ }^{19}$ This is crucial since it has been widely demonstrated that not only chronic NHE-1 hyperactivity plays a key role in pathological cardiac hypertrophy development but also its specific inhibition is sufficient to induce hypertrophy regression. ${ }^{4,24,27-31}$ The main mechanism involved would be the cytoplasmic sodium overload that reduces forward and favors reverse mode of the sarcoplasmic $\mathrm{Na}^{+} / \mathrm{Ca}^{2+}$ exchanger increasing cytoplasmic calcium and as a consequence calcineurin activation, among other prohypertrophic molecules. ${ }^{29}$ Another important consequence of NHE-1 hyperactivity in the setup of pathologic cardiac hypertrophy and heart failure would be the decrease in mitochondrial calcium (again due to cytoplasmic sodium overload) that favors oxidative stress and impairs ATP synthesis. ${ }^{32}$ On the other hand, NHE-1 acute activation in response to intracellular acidosis is crucial in order to keep intracellular $\mathrm{pH}$ in the physiologic range, even more during high metabolic states such as exercise. In this sense, it is important to highlight that under physiological hemodynamic overload, i.e. endurance training, despite the myocardium is stretched, NHE-1 is not hyperactive but preserves its function to participate in steady-state intracellular $\mathrm{pH}$ regulation. ${ }^{19}$ Interestingly, in the 
present work we demonstrate that IGF-1 is also capable of impeding NHE-1 hyperactivity in the hypertrophied cardiomyocytes of the SHR. This effect depends on the activation of the IGF-1R and the PI-3K/AKT pathway leading to the inhibitory phosphorylation of the residue Ser648 in the regulatory domain of the exchanger.

Based on our previous results unveiling an improvement in cardiac function in the swimming-trained $\mathrm{SHR}^{8}$ we evaluated the acute effect of IGF-1 upon contractility in hypertrophied SHR isolated cardiomyocytes. We were able to identify a significant improvement in contraction as well as in relaxation. This data is in agreement with similar findings already reported for both normal and failing cardiomyocytes, ${ }^{18,33-36}$ but are in conflict with the results of Ren et al. ${ }^{37}$ These last authors were not able to detect a positive inotropic effect of IGF-1 in SHR cardiomyocyte, although they did it in cardiomyocytes from normotensive rats, suggesting the existence of an IGF-1 resistant state in the SHR hearts. However, it has been repeatedly reported the upregulation of the IGF-1R, both at the mRNA and protein level in various models of hypertension, including human essential hypertension. ${ }^{38-40}$

With respect to the mechanism involved in the acute positive inotropic/lusitropic effect of IGF-1, there are some results from others and our own group already published, although contradictory. It has been proposed both an increase in calcium availability, ${ }^{18,35}$ as well as the opposite situation: conserved calcium transients with improved myofilament sensitivity. ${ }^{41}$ Under the experimental conditions of our work, we were able to detect an increase in calcium transient amplitude and rate of decay, probably due to an improvement in SERCA 2a function secondary to the CaMKIIphosphorylation of PLN. In the setup of chronic treatment with IGF-1 the increases in contraction and relaxation were attributed to an up-regulation of SERCA $2 a$ 
expression. ${ }^{36}$ This cannot be the case in our experiments, due to the short incubation time with IGF-1 (15 min) used.

Common to cardiovascular diseases, including hypertensive cardiac hypertrophy, is increased oxidant stress, characterized by the spatio-temporal imbalance between the production of ROS and/or reactive nitrogen species and the detoxification through enzymatic and non-enzymatic systems. Despite the fact that limited levels of these molecules are necessary for several physiological processes, ${ }^{42,}{ }^{43}$ studies using cell culture and experimental animal models clearly support the role of oxidant stress in the onset and progression of cardiac diseases. In the present work, and in agreement with previous reports (for review see ${ }^{44}$ ), we were able to detect an antioxidant effect of IGF-1 evidenced by a decrease in cardiac $\mathrm{H}_{2} \mathrm{O}_{2}$ accumulation and a higher SOD activity. Further research will be necessary to elucidate the subcellular mechanism involved in this antioxidant effect of IGF-1. Regarding to SOD activity, an AKT-dependent post-translational modification of the enzyme seems to play a key role under the experimental conditions assayed by us. Interestingly, by using the group-based phosphorylation predicting and scoring method we found potential AKT phosphorylation consensus sites in SOD. ${ }^{45}$ In line with our results are those obtained by Borges et al. who found that exercise training increases SOD activity by a post-translational mechanism in hearts from exercised Wistar rats. ${ }^{46}$ Another mechanism that could be probably contributing to the decrease in oxidative stress is the prevention of intracellular sodium overload by the inhibition of NHE-1 hyperactivity preserving mitochondrial calcium, as it has been recently reported by Baartscheer and colleagues. ${ }^{32}$ The latter is considered to be an important activator of ATP synthesis and of the antioxidant enzymatic network. ${ }^{47,48}$

This article is protected by copyright. All rights reserved. 
Several potential limitations should be acknowledged in this study. First, the concentration used of IGF-1 used appears to be relatively high compared to the plasma concentration (15-30 $\mathrm{ng} \mathrm{ml}$-1). However, the myocardium itself produces IGF-1 possibly leading to higher local concentrations. Second, we did not explore the exact mechanism responsible for the reduction in $\mathrm{H}_{2} \mathrm{O}_{2}$. Based on previous results from our group, in which NHE-1 inhibition reduced mitochondrial ROS production, ${ }^{23}$ we think this as the most probable explanation. However, future experiments will be necessary to elucidate this point. Last but not least, further insight will be necessary to clarify the intracellular events contributing to the positive inotropic/lusitropic effect of IGF-1.

\section{MATERIAL AND METHODS}

Male SHRs at 4 months of age (body weight: $300-400 \mathrm{~g}$ ) were anesthetized by intraperitoneal injection of ketamine (50 mg kg-1 of body weight) diazepam (2.5 mg kg-1 of body weight) and hearts rapidly excised when plane 3 of phase III of anaesthesia was reached.

All procedures followed during this investigation conform to the to the 'European Convention for the Protection of Vertebrate Animals used for Experimental and other Scientific Purposes' (Council of Europe No 123, Strasbourg 1985) and the experimental protocol was approved by the Animal Welfare Committee of La Plata School of Medicine.

\subsection{Heart perfusion, cell isolation and culture:}

Hearts were retrograde perfused with a $\mathrm{CO}_{2} / \mathrm{HCO}_{3}{ }^{-}$-buffered solution equilibrated with $5 \% \mathrm{CO}_{2}-95 \% \mathrm{O}_{2}$ at $37{ }^{\circ} \mathrm{C}(\mathrm{pH} \sim 7.40)$, during 15 min under the presence or 
absence of IGF-1, as previously described. ${ }^{19}$ Pharmacologic inhibitors were added 5 minutes before IGF-1, when indicated.

Rat ventricular myocytes were isolated from hearts mounted in a Langendorff apparatus and then suspended in supplemented medium to attach to glass coverslips pretreated with laminin as previously described. ${ }^{19}$ Unattached myocytes were removed by gently changing the medium. The new medium was the same than the previous one except for being serum deprived, and, when corresponding, supplemented with $10 \mathrm{nmol}$ L-1 IGF-1. The different antagonists used were added to the serum-deprived medium 10 minutes before IGF-1. Cardiomyocytes were kept in culture for $24 \mathrm{~h}$.

\subsection{NHE-1 Activity}

Intracellular $\mathrm{pH}\left(\mathrm{pH}_{\mathrm{i}}\right)$ was recorded in single myocytes maintained in $\mathrm{HCO}_{3}^{-}$free solution following the previously described BCECF epifluorescence technique. ${ }^{19,} 49$ The ammonium pre-pulse (20 mmol L-1 $\mathrm{NH}_{4} \mathrm{Cl}$ for $3 \mathrm{~min}$ )-induced acid load method was used to calculate proton efflux $\left(\mathrm{J}_{\mathrm{H}}\right)$ in order to evaluate NHE-1 activity following a protocol already described. ${ }^{19}$

\subsection{Real time RT-PCR}

NHE-1 mRNA expression normalized to GAPDH was measured by real-time RTPCR in cultured cardiac myocytes following a procedure previously described. ${ }^{50}$ The following primers were used: 5'-GGGTGTGAACCACGAGAAAT-3' (forward) and 5'CCACAGTCTTCTGAGTGGCA-3' (reverse) for GAPDH; 5'TCACCACAGCTCCATCAGAG-3' (forward) and 5'- GATCTCGAAGGGTGTCCGTA3' (reverse). 


\subsection{Western Blotting}

Myocardial samples from the left ventricles were homogenized according to a procedure previously described. ${ }^{19}$ For the immunodetection, the following specific antibodies were used: anti phospho-ERK1/2 (Santa Cruz Biotechnology, sc-16982), anti phospho-AKT (Cell Signaling, 4060S), anti phospho-p90 ${ }^{\text {RSK }}$ (Cell Signaling \#9341), anti NHE-1 (Santa Cruz Biotechnology sc-28758), anti phospho-Thr286CaMKII (P-CaMKII, 1:1000; Abcam), anti phospho-Thr17-PLN (2:500; Badrilla) and anti phospho-Ser16-PLN (2:500; Millipore). Phosphorylation at Ser703 or Ser648 was estimated in immunoprecipitated NHE-1 incubated with anti-P-14-3-3 binding motif antibody (Cell Signaling, \#9601) or anti-P-AKT substrate (Cell Signaling, \#10001S) as previously described. ${ }^{24,26}$ IGF-1R phosphorylation was determined in immunoprecipitated samples as previously described. ${ }^{51}$ The detection of GAPDH (Millipore MAB374) was used as a loading control for the immunoblots except when the phosphorylation of NHE-1 or IGF-1R was being evaluated in immunoprecipitated samples. In these particular cases, the total amount of each specific protein immunoprecipitated (phosphorylated and unphosphorylated) was use as the loading control. The secondary antibodies used were peroxidase-conjugated anti-rabbit (NA934, GE Healthcare Life Sciences), anti-mouse (NA931, GE Healthcare Life Sciences) or anti-goat IgG (sc-2004). Bands were visualized and analyzed as previously. $^{19}$

\subsection{Contractility/Relaxation and Calcium transients}

The effect of IGF-1 on contractility was explored in ventricular myocytes superfused with a solution containing $1 \mathrm{mmol} \mathrm{L}-1 \mathrm{CaCl}_{2}, \mathrm{pH} 7.4$ with continuous bubbling with $1 \% \mathrm{CO}_{2}$ and $99 \% \mathrm{O}_{2}$ at $30{ }^{\circ} \mathrm{C}$ in an inverted microscope (Nikon TE 2000-U). Myocytes were stimulated via two-platinum electrodes on either side of the bath at 
$0.5 \mathrm{~Hz}$. The myocytes were observed using a video-camera connected to the microscope and sarcomere length (SL) was recorded by specific software (ION WIZARD analysis software). Contractility was measured in a limited region of the myocyte and the software estimated the most frequent $S L$ in that region using fast Fourier transforms analysis (Ion Optix, Milton, MA). Time constant of twitch decay (tau) was measured as an indication of relaxation. Myocytes were allowed to contract over 5 minutes to ensure steady state before superfusion with IGF-1.

A previously described protocol was follow to measure intracellular calcium measurements in isolated cardiomyocytes loaded with $10 \mu \mathrm{mol} / \mathrm{L}$ Fura-2 AM (Molecular probes). ${ }^{18}$ When corresponding, inhibitors were applied to the cells 5 minutes before IGF-1 addition. Results were expressed as a percentage of time cero (immediately before IGF-1 addition).

\subsection{ROS production}

ROS production was measured in freshly isolated cardiomyocytes loaded with 10 umol L-1 5- (and-6)-chloromethyl-2',7'-dichlorodihydrofluorescein diacetate, acetyl ester (CM-H2DCFDA, Invitrogen) as previously reported. ${ }^{18}$ Briefly, cardiomyocytes were excited at $495 \mathrm{~nm}$ and emission spectra was acquired through a $510 \mathrm{~nm}$ filter with a Nikon camera attached to an inverted Zeiss LSM 410 microscope. During 20 min, photographs were taken every 30 seconds in the different experimental groups. When indicated, the inhibitors were administrated 5 min before IGF-1. Results were analyzed with Image $\mathrm{J}$ software and expressed as the ratio between the slope after/before IGF-1.

This article is protected by copyright. All rights reserved. 


\subsection{Superoxide dismutase (SOD)activity}

SOD activity was determined by inhibition of formazan production (produced by nitroblue tetrazolium [NBT] reduction by superoxide anion) at $\mathrm{pH} 10.2$ and $25^{\circ} \mathrm{C}$ as

described previously. ${ }^{22}$ The reaction was started by the addition of xanthine oxidase, and then the absorbance at $560 \mathrm{~nm}$ was read every 10 seconds for 120 seconds. One unit of SOD was defined as the amount of enzymatic protein required to inhibit $50 \%$ of NBT reduction.

\subsection{Chemicals}

All drugs used in the present study were analytical reagent. IGF-1 (10 nmol L-1): recombinant mouse insulin like growth factor -1 from Gibco by Life Technologies (Cat \# PMG0075); AG1024 (100 nmol L-1): IGF-1R antagonist from Santa Cruz Biotechnology (Cat \# sc-205907); MK2206 (500 nmol L-1): AKT inhibitor from Selleck Chemicals (Cat \# S1078); wortmannin (100 nmol L-1): PI3K inhibitor from Sigma Argentina (Cat \# W1628). Wortmannin, MK2206 and AG1024 were diluted in DMSO (final concentration 0.01\%).

\subsection{Statistics}

Results are expressed as mean \pm SEM. The Student $t$ test, 1-way ANOVA followed by the Student-Newman-Keuls test or two-way ANOVA were used when corresponding. Significance level was set at $p<0.05$.

\section{ACKNOWLEDGEMENTS}

This work was supported in part by grant PICT 2012- 2907, PICT 2012-0578 from Agencia Nacional de Promoción Científica y Tecnológica de Argentina; PIP 0433 
from Consejo Nacional de Investigaciones Científicas y Tecnológicas (CONICET)

and M11/165 from Universidad Nacional de La Plata to Dr. Irene L. Ennis.

We specially thank Erica Pereyra for technical assistance.

\section{CONFLICT OF INTEREST}

None.

\section{REFERENCES}

1. Wakabayashi S, Hisamitsu T and Nakamura TY. Regulation of the cardiac $\mathrm{Na}(+) / \mathrm{H}(+)$ exchanger in health and disease. J Mol Cell Cardiol. 2013;61:68-76.

2. Cingolani HE and Ennis IL. Sodium-hydrogen exchanger, cardiac overload, and myocardial hypertrophy. Circulation. 2007;115:1090-100.

3. Nakamura TY, Iwata Y, Arai Y, Komamura K and Wakabayashi S. Activation of $\mathrm{Na}+/ \mathrm{H}+$ exchanger 1 is sufficient to generate $\mathrm{Ca} 2+$ signals that induce cardiac hypertrophy and heart failure. Circ Res. 2008;103:891-9.

4. Nolly MB, Pinilla AO, Ennis IL, Cingolani HE and Morgan PE. Cardiac hypertrophy reduction in SHR by specific silencing of myocardial $\mathrm{Na}(+) / \mathrm{H}(+)$ exchanger. J Appl Physiol (1985). 2015;118:1154-

60.

5. Perez NG, Alvarez BV, Camilion de Hurtado MC and Cingolani HE. pHi regulation in myocardium of the spontaneously hypertensive rat. Compensated enhanced activity of the $\mathrm{Na}(+)-\mathrm{H}+$ exchanger. Circ Res. 1995;77:1192-200.

6. Schussheim AE and Radda GK. Altered $\mathrm{Na}(+)-\mathrm{H}(+)$ - exchange activity in the spontaneously hypertensive perfused rat heart. J Mol Cell Cardiol. 1995;27:1475-81.

7. Cingolani HE, Ennis IL, Aiello EA and Perez NG. Role of autocrine/paracrine mechanisms in response to myocardial strain. Pflugers Arch. 2011;462:29-38.

8. Garciarena CD, Pinilla OA, Nolly MB, Laguens RP, Escudero EM, Cingolani HE and Ennis IL. Endurance training in the spontaneously hypertensive rat: conversion of pathological into physiological cardiac hypertrophy. Hypertension. 2009;53:708-14.

9. Scheinowitz M, Kessler-Icekson G, Freimann S, Zimmermann R, Schaper W, Golomb E, Savion $\mathrm{N}$ and Eldar $\mathrm{M}$. Short- and long-term swimming exercise training increases myocardial insulin-like growth factor-I gene expression. Growth Horm IGF Res. 2003;13:19-25.

10. Neri Serneri GG, Boddi M, Modesti PA, Cecioni I, Coppo M, Padeletti L, Michelucci A, Colella $A$ and Galanti G. Increased cardiac sympathetic activity and insulin-like growth factor-I formation are associated with physiological hypertrophy in athletes. Circ Res. 2001;89:977-82.

11. Poehlman ET, Rosen $\mathrm{CJ}$ and Copeland KC. The influence of endurance training on insulin-like growth factor-1 in older individuals. Metabolism. 1994;43:1401-5.

12. McMullen JR, Shioi T, Huang WY, Zhang L, Tarnavski O, Bisping E, Schinke M, Kong S, Sherwood MC, Brown J, Riggi L, Kang PM and Izumo S. The insulin-like growth factor 1 receptor

This article is protected by copyright. All rights reserved. 
induces physiological heart growth via the phosphoinositide 3-kinase(p110alpha) pathway. J Biol Chem. 2004;279:4782-93.

13. Kim J, Wende AR, Sena S, Theobald HA, Soto J, Sloan C, Wayment BE, Litwin SE, Holzenberger $M$, LeRoith $D$ and Abel ED. Insulin-like growth factor I receptor signaling is required for exerciseinduced cardiac hypertrophy. Mol Endocrinol. 2008;22:2531-43.

14. Troncoso R, Ibarra C, Vicencio JM, Jaimovich E and Lavandero S. New insights into IGF-1 signaling in the heart. Trends Endocrinol Metab. 2014;25:128-37.

15. DeBosch B, Treskov I, Lupu TS, Weinheimer C, Kovacs A, Courtois M and Muslin AJ. Akt1 is required for physiological cardiac growth. Circulation. 2006;113:2097-104.

16. McMullen JR, Shioi T, Zhang L, Tarnavski O, Sherwood MC, Kang PM and Izumo S.

Phosphoinositide 3-kinase(p110alpha) plays a critical role for the induction of physiological, but not pathological, cardiac hypertrophy. Proc Natl Acad Sci U S A. 2003;100:12355-60.

17. Shioi T, Kang PM, Douglas PS, Hampe J, Yballe CM, Lawitts J, Cantley LC and Izumo S. The conserved phosphoinositide 3-kinase pathway determines heart size in mice. EMBO J. 2000;19:253748.

18. Burgos JI, Yeves AM, Barrena JP, Portiansky EL, Vila-Petroff MG and Ennis IL. Nitric oxide and CaMKII: Critical steps in the cardiac contractile response To IGF-1 and swim training. J Mol Cell Cardiol. 2017;112:16-26.

19. Yeves AM, Villa-Abrille MC, Perez NG, Medina AJ, Escudero EM and Ennis IL. Physiological cardiac hypertrophy: Critical role of AKT in the prevention of NHE-1 hyperactivity. J Mol Cell Cardiol. 2014;76C:186-195.

20. Takimoto E and Kass DA. Role of oxidative stress in cardiac hypertrophy and remodeling. Hypertension. 2007;49:241-8.

21. Sorescu D and Griendling KK. Reactive oxygen species, mitochondria, and NAD(P)H oxidases in the development and progression of heart failure. Congest Heart Fail. 2002;8:132-40.

22. Alvarez MC, Caldiz C, Fantinelli JC, Garciarena CD, Console GM, Chiappe de Cingolani GE and Mosca SM. Is cardiac hypertrophy in spontaneously hypertensive rats the cause or the consequence of oxidative stress? Hypertens Res. 2008;31:1465-76.

23. Garciarena CD, Caldiz Cl, Correa MV, Schinella GR, Mosca SM, Chiappe de Cingolani GE, Cingolani HE and Ennis IL. Na+/H+ exchanger-1 inhibitors decrease myocardial superoxide production via direct mitochondrial action. J Appl Physiol. 2008;105:1706-13.

24. Garciarena CD, Caldiz Cl, Portiansky EL, Chiappe de Cingolani GE and Ennis IL. Chronic NHE-1 blockade induces an antiapoptotic effect in the hypertrophied heart. J Appl Physiol. 2009;106:132531.

25. Takahashi E, Abe J, Gallis B, Aebersold R, Spring DJ, Krebs EG and Berk BC. p90(RSK) is a serum-stimulated $\mathrm{Na}+\mathrm{H}+$ exchanger isoform-1 kinase. Regulatory phosphorylation of serine 703 of $\mathrm{Na}+/ \mathrm{H}+$ exchanger isoform-1.J Biol Chem. 1999;274:20206-14.

26. Snabaitis AK, Cuello F and Avkiran M. Protein kinase B/Akt phosphorylates and inhibits the cardiac $\mathrm{Na}+/ \mathrm{H}+$ exchanger NHE1. Circ Res. 2008;103:881-90.

27. Dulce RA, Hurtado C, Ennis IL, Garciarena CD, Alvarez MC, Caldiz C, Pierce GN, Portiansky EL, Chiappe de Cingolani GE and Camilion de Hurtado MC. Endothelin-1 induced hypertrophic effect in neonatal rat cardiomyocytes: involvement of $\mathrm{Na}+/ \mathrm{H}+$ and $\mathrm{Na}+/ \mathrm{Ca} 2+$ exchangers. J Mol Cell Cardiol. 2006;41:807-15.

28. Ennis IL, Escudero EM, Console GM, Camihort G, Dumm CG, Seidler RW, Camilion de Hurtado $\mathrm{MC}$ and Cingolani HE. Regression of isoproterenol-induced cardiac hypertrophy by $\mathrm{Na}+\mathrm{H}+$ exchanger inhibition. Hypertension. 2003;41:1324-9.

29. Ennis IL, Garciarena CD, Escudero EM, Perez NG, Dulce RA, Camilion de Hurtado MC and Cingolani HE. Normalization of the calcineurin pathway underlies the regression of hypertensive hypertrophy induced by $\mathrm{Na}+/ \mathrm{H}+$ exchanger-1 (NHE-1) inhibition. Can J Physiol Pharmacol.

2007;85:301-10.

This article is protected by copyright. All rights reserved. 
30. Hisamitsu T, Nakamura TY and Wakabayashi S. $\mathrm{Na}(+) / \mathrm{H}(+)$ exchanger 1 directly binds to calcineurin A and activates downstream NFAT signaling, leading to cardiomyocyte hypertrophy. Mol Cell Biol. 2012;32:3265-80.

31. Baartscheer A, Hardziyenka M, Schumacher CA, Belterman CN, van Borren MM, Verkerk AO, Coronel R and Fiolet JW. Chronic inhibition of the $\mathrm{Na}+\mathrm{H}+-$ exchanger causes regression of hypertrophy, heart failure, and ionic and electrophysiological remodelling. Br J Pharmacol. 2008;154:1266-75.

32. Baartscheer A, Schumacher CA, Wust RC, Fiolet JW, Stienen GJ, Coronel R and Zuurbier CJ. Empagliflozin decreases myocardial cytoplasmic $\mathrm{Na}(+)$ through inhibition of the cardiac $\mathrm{Na}(+) / \mathrm{H}(+)$ exchanger in rats and rabbits. Diabetologia. 2017;60:568-573.

33. Donath MY, Sutsch G, Yan XW, Piva B, Brunner HP, Glatz Y, Zapf J, Follath F, Froesch ER and Kiowski W. Acute cardiovascular effects of insulin-like growth factor I in patients with chronic heart failure. J Clin Endocrinol Metab. 1998;83:3177-83.

34. Thuesen L, Christiansen JS, Sorensen KE, Jorgensen JO, Orskov $\mathrm{H}$ and Henningsen $\mathrm{P}$. Increased myocardial contractility following growth hormone administration in normal man. An echocardiographic study. Dan Med Bull. 1988;35:193-6.

35. Kinugawa S, Tsutsui H, Ide T, Nakamura R, Arimura K, Egashira K and Takeshita A. Positive inotropic effect of insulin-like growth factor-1 on normal and failing cardiac myocytes. Cardiovasc Res. 1999;43:157-64.

36. Kim SJ, Abdellatif M, Koul S and Crystal GJ. Chronic treatment with insulin-like growth factor I enhances myocyte contraction by upregulation of Akt-SERCA2a signaling pathway. Am J Physiol Heart Circ Physiol. 2008;295:H130-5.

37. Ren J, Jefferson L, Sowers JR and Brown RA. Influence of age on contractile response to insulin-like growth factor 1 in ventricular myocytes from spontaneously hypertensive rats. Hypertension. 1999;34:1215-22.

38. Delafontaine P. Insulin-like growth factor I and its binding proteins in the cardiovascular system. Cardiovasc Res. 1995;30:825-34.

39. Guron G, Friberg P, Wickman A, Brantsing C, Gabrielsson B and Isgaard J. Cardiac insulin-like growth factor I and growth hormone receptor expression in renal hypertension. Hypertension. 1996;27:636-42.

40. Diez J and Laviades $\mathrm{C}$. Insulin-like growth factor-1 and cardiac mass in essential hypertension: comparative effects of captopril, lisinopril and quinapril. J Hypertens Suppl. 1994;12:S31-6.

41. Bathgate-Siryk A, Dabul S, Pandya K, Walklett K, Rengo G, Cannavo A, De Lucia C, Liccardo D, Gao E, Leosco D, Koch WJ and Lymperopoulos A. Negative impact of beta-arrestin-1 on postmyocardial infarction heart failure via cardiac and adrenal-dependent neurohormonal mechanisms. Hypertension. 2014;63:404-12.

42. Droge W. Free radicals in the physiological control of cell function. Physiol Rev. 2002;82:4795.

43. Wang W, Fang $\mathrm{H}$, Groom L, Cheng A, Zhang W, Liu J, Wang X, Li K, Han P, Zheng M, Yin J, Wang W, Mattson MP, Kao JP, Lakatta EG, Sheu SS, Ouyang K, Chen J, Dirksen RT and Cheng H. Superoxide flashes in single mitochondria. Cell. 2008;134:279-90.

44. Campos JC, Gomes KM and Ferreira JC. Impact of exercise training on redox signaling in cardiovascular diseases. Food Chem Toxicol. 2013;62:107-19.

45. Zhou FF, Xue Y, Chen GL and Yao X. GPS: a novel group-based phosphorylation predicting and scoring method. Biochem Biophys Res Commun. 2004;325:1443-8.

46. Borges JP, Verdoorn KS, Daliry A, Powers SK, Ortenzi VH, Fortunato RS, Tibirica E and Lessa MA. Delta opioid receptors: the link between exercise and cardioprotection. PLoS One. 2014;9:e113541.

47. Liu T, Takimoto E, Dimaano VL, DeMazumder D, Kettlewell S, Smith G, Sidor A, Abraham TP and O'Rourke B. Inhibiting mitochondrial $\mathrm{Na}+/ \mathrm{Ca} 2+$ exchange prevents sudden death in a Guinea pig model of heart failure. Circ Res. 2014;115:44-54.

This article is protected by copyright. All rights reserved. 
48. Kohlhaas M, Liu T, Knopp A, Zeller T, Ong MF, Bohm M, O'Rourke B and Maack C. Elevated cytosolic $\mathrm{Na}+$ increases mitochondrial formation of reactive oxygen species in failing cardiac myocytes. Circulation. 2010;121:1606-13.

49. Yeves AM, Garciarena CD, Nolly MB, Chiappe de Cingolani GE, Cingolani HE and Ennis IL. Decreased activity of the $\mathrm{Na}+\mathrm{H}+$ exchanger by phosphodiesterase $5 \mathrm{~A}$ inhibition is attributed to an increase in protein phosphatase activity. Hypertension. 2010;56:690-5.

50. Ennis IL, Garciarena CD, Perez NG, Dulce RA, Camilion de Hurtado MC and Cingolani HE. Endothelin isoforms and the response to myocardial stretch. Am J Physiol Heart Circ Physiol. 2005;288:H2925-30.

51. Ikeda H, Shiojima I, Ozasa Y, Yoshida M, Holzenberger M, Kahn CR, Walsh K, Igarashi T, Abel ED and Komuro I. Interaction of myocardial insulin receptor and IGF receptor signaling in exerciseinduced cardiac hypertrophy. J Mol Cell Cardiol. 2009;47:664-75.

This article is protected by copyright. All rights reserved. 


\section{LEGENDS TO FIGURES}

Figure 1. The NHE-1 is hyperactive in the myocardium of the SHR due to a posttranslational modification. (A) Average results of NHE-1 activity assessed as proton efflux $\left(\mathrm{JH}_{\mathrm{H}}^{+}\right)$during the recovery from intracellular acidosis in isolated ventricular myocytes of adult SHR and Wistar rats. These experiments were performed in bicarbonate free medium in order to exclude the involvement of any other alkalinizing mechanism. (B) No significant difference in myocardial NHE-1 expression between SHR and Wistar rats was detected by immunoblots. (C) A higher phosphorylation of the stimulatory residue serine 703 (downstream target of ERK1/2 pathway) was observed in SHR while no difference was evident in the inhibitory residue serine 648 (downstream target of AKT) of the NHE-1 regulatory tail between rat strains. (D) As expected, a greater activation exclusively of the ERK1/2 pathway was evident in the SHR myocardium compared to Wistar. Data are express as mean \pm SEM. Representative immunoblots are shown on top of bars when corresponding. The number of experiments $(n)$ is depicted between brackets. * indicates $\mathrm{P}<0.05$ vs. Wistar rats (control), t-test.

Figure 2. IGF-1 exerts an AKT-dependent inhibitory effect on NHE-1 activity in SHR cardiomyocytes. (A) Representative recordings of $\mathrm{pHi}$ vs. time in the absence (control), presence of $10 \mathrm{nmol} / \mathrm{L}$ IGF-1, or exposed to $10 \mathrm{nmol} / \mathrm{L}$ IGF-1 in combination with AG1024 (AG, specific antagonist of IGF-1R), wortmannin (Wort, inhibitor of PI3K/AKT) or MK2206 (MK, specific inhibitor of AKT). (B) Average results for NHE-1 activity measured as proton efflux $\left(\mathrm{J}^{+}\right)$. Comparison among groups was done at a common intracellular $\mathrm{pH}$ of 6.95 , as explained in Methods. It can be 
appreciated the inhibitory effect of IGF-1 that was cancelled by the different pharmacologic inhibitors assayed. * indicates $\mathrm{P}<0.05$ vs. control, ANOVA. (C) IGF-1 did not modified NHE-1 expression evaluated by quantitative RT-PCR. (D) No significant difference was detected in intrinsic buffer capacity $(\beta i)$ in the presence of IGF-1. The number of experiments (n) is depicted between brackets. * indicates $\mathrm{P}<0.05$ vs. control (absence of IGF-1), t-test.

Figure 3. Signaling pathway activated by IGF-1 in the SHR myocardium. IGF-1 stimulated the phosphorylation, and therefore activation, of its own receptor (IGF1R), AKT, ERK1/2 and p90RSK; being these effects blunted by AG1024, a specific antagonist of IGF-1R (Panels A, B, C and D, respectively). (E) In agreement with these results, a significant increase in the phosphorylation of the AKT-dependent inhibitory site (serine 648) of the cytosolic regulatory domain of the NHE-1 was detected. However, no differences at the stimulatory serine 703 site, a specific target of ERK1/2-p90RSK pathway, were evident, probably because this site is already hyperphosphorylated in the SHR myocardium. Representative immunoblots are shown on top and average data \pm SEM on bottom of each panel. The number of experiments $(n)$ is depicted between brackets. * indicates $P<0.05$ vs. control (absence of IGF-1), ANOVA.

Figure 4. IGF-1 improves contractility in SHR cardiomyocytes. (A) Representative continuous recordings of sarcomere shortening (left) and individual traces before (a) and after (b) IGF-1 (right) measured in SHR isolated cardiomyocytes. (B) Average results of the time course of sarcomere shorting for each group. (C) Average values of the difference in the relaxation time constant -Tau- at 15 minutes of the experimental protocol for each group. AG: AG1024, specific antagonist of IGF-1R; MK: MK2206, specific AKT inhibitor. Average data are express as mean \pm SEM. The 
number of experiments $(n)$ is depicted between brackets. ${ }^{*}$ indicates $P<0.05$ vs. control (absence of IGF-1), ANOVA.

Figure 5. IGF-1 increases calcium transient amplitude and rate of decay in SHR isolated cardiomyocytes. (A) Typical tracings showing the effect of IGF-1 on the amplitude and decay of the calcium transient. (B) - (C) Overall results for the amplitude and time to $50 \%$ decay of the calcium transient for each experimental group, respectively. (D) Representative Western blots and average results showing the effect of IGF-1 on the phosphorylation of CaMKII; threonine 17 (Thr17-PLN, target of CaMKII) and serine 16 of phospholamban (Ser16-PLN, target of PKA) in the absence and presence of AG1024 (AG). The number of experiments (n) is depicted between brackets. ${ }^{*}$ indicates $\mathrm{P}<0.05$ vs. control (absence of IGF-1), ANOVA.

Figure 6. IGF-1 has an antioxidant effect on the SHR myocardium. (A) Typical recordings of DCFDA fluorescence in isolated SHR cardiomyocytes. (B) Average results of $\mathrm{H}_{2} \mathrm{O}_{2}$ production estimated by the ratio between the slope 2 (after) and slope 1 (before) the exposure to IGF-1. (C) Representative measurements and (D) average results of myocardial superoxide dismutase (SOD) activity. The experiments were performed under control conditions or in the presence of IGF-1 with/without the IGF-1R antagonist AG1024 (AG) or the AKT specific inhibitor MK2206. Average data are express as mean \pm SEM. The number of experiments $(n)$ is depicted between brackets. * indicates $\mathrm{P}<0.05$ vs. control (absence of IGF-1), ANOVA.

Figure 7. Cardioprotective effects of IGF-1 on the SHR myocardium. This cartoon schematically summarizes a subcellular explanation for the beneficial effects of IGF1 , and therefore probably for exercise training, on the hypertrophied heart of the SHR. IGF-1 by stimulating its specific receptor (IGF-1R) activates PI3K/AKT 
signaling that prevents NHE-1 hyperactivity and improves cardiac contractility and oxidative stress.

A

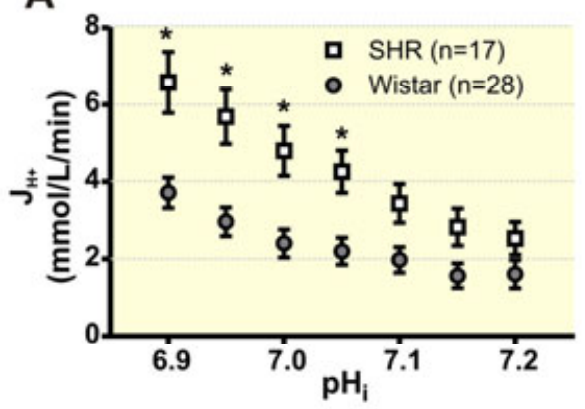

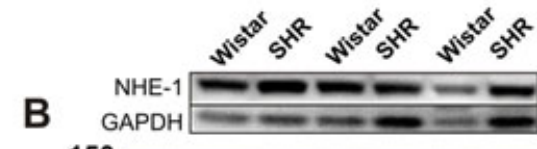

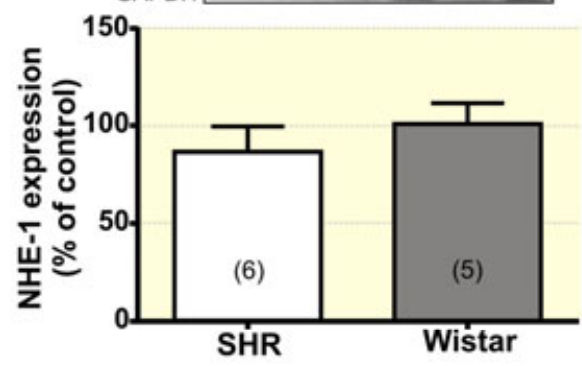

D
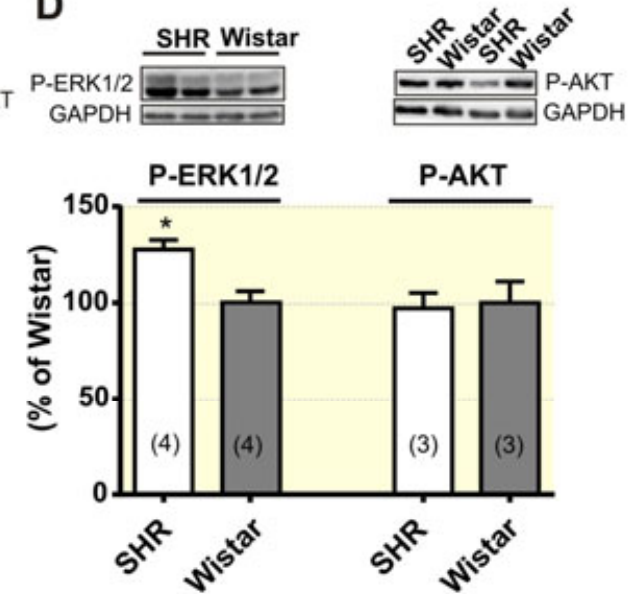

This article is protected by copyright. All rights reserved. 

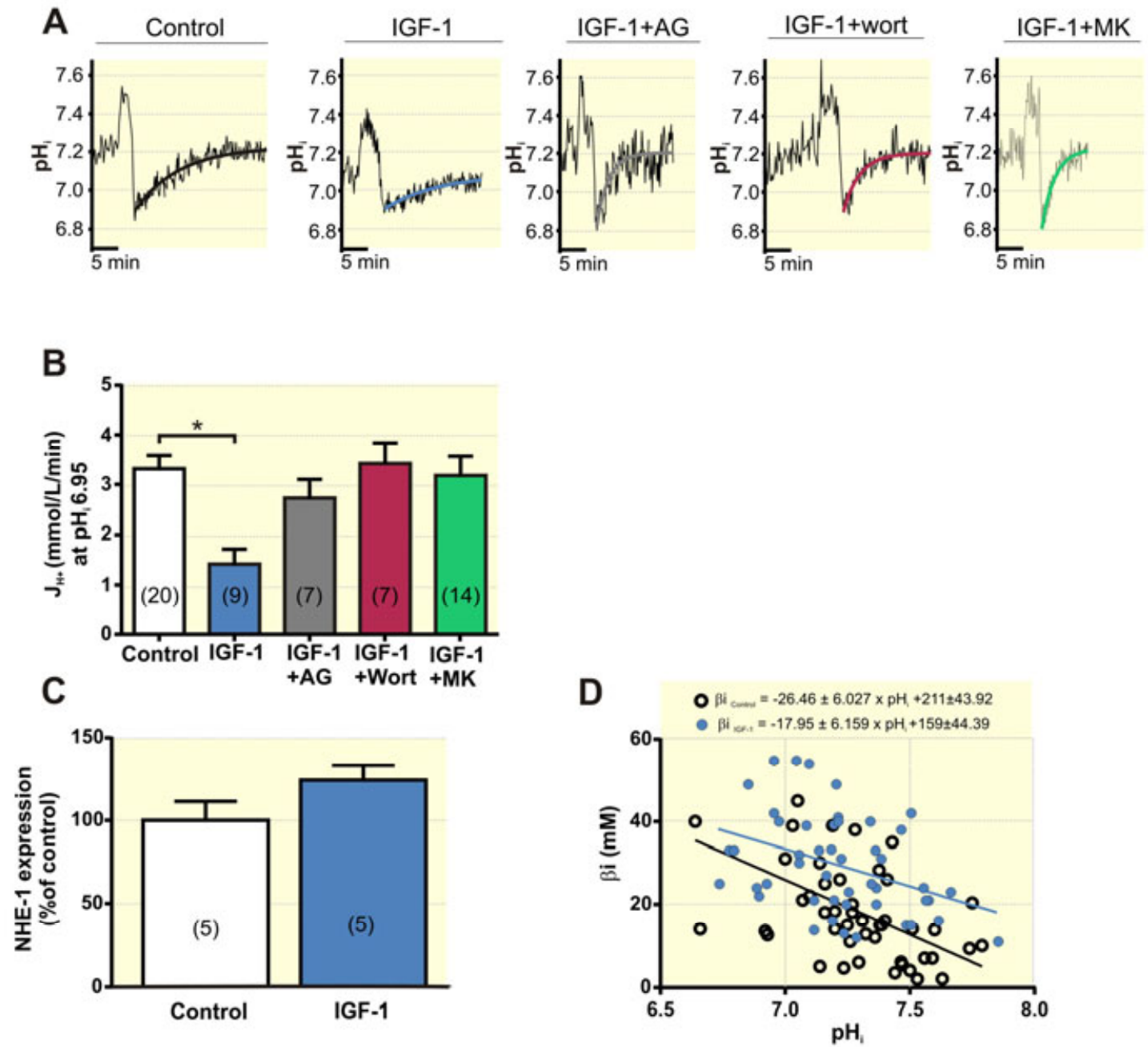

This article is protected by copyright. All rights reserved. 

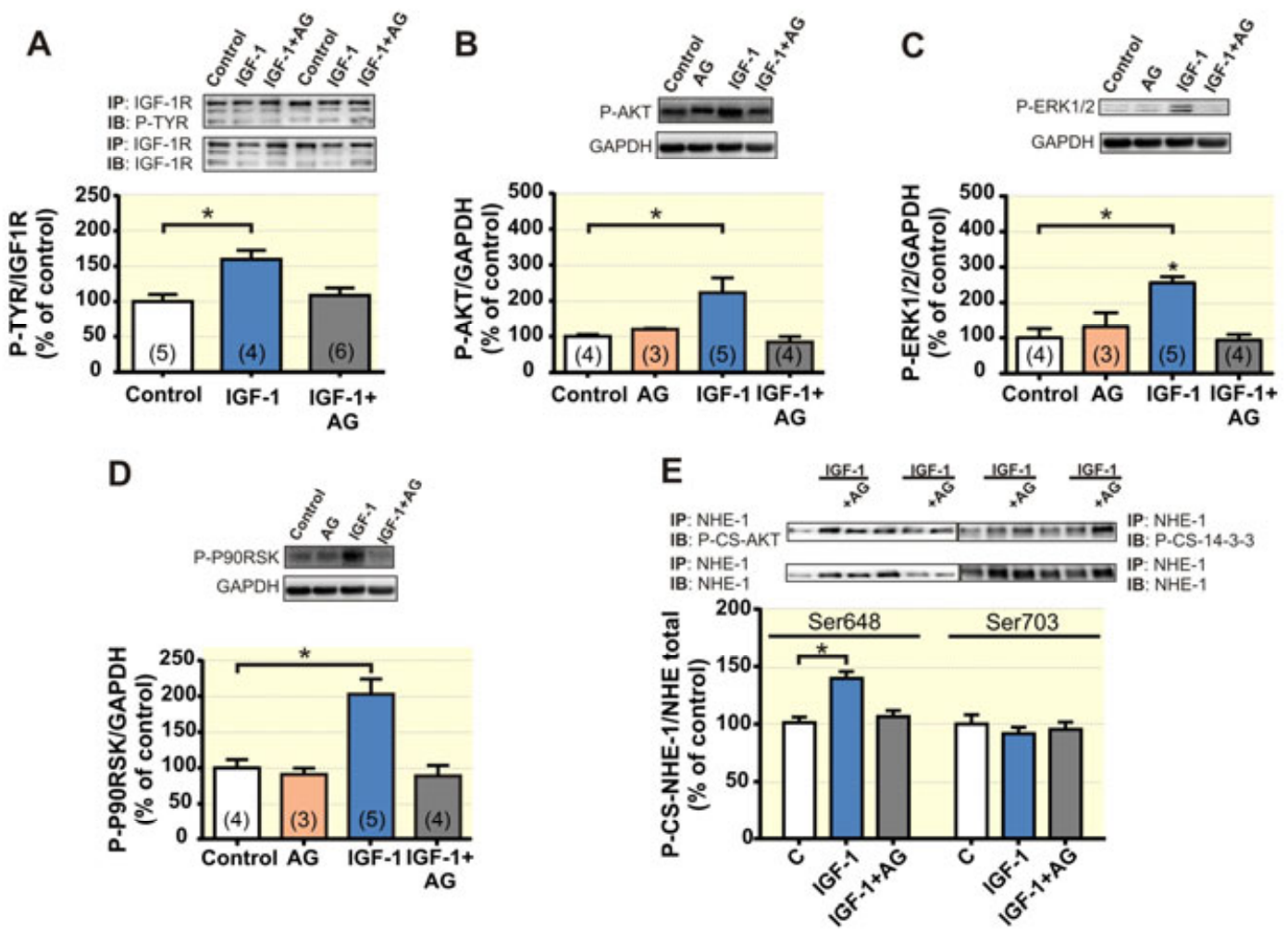

This article is protected by copyright. All rights reserved. 
A
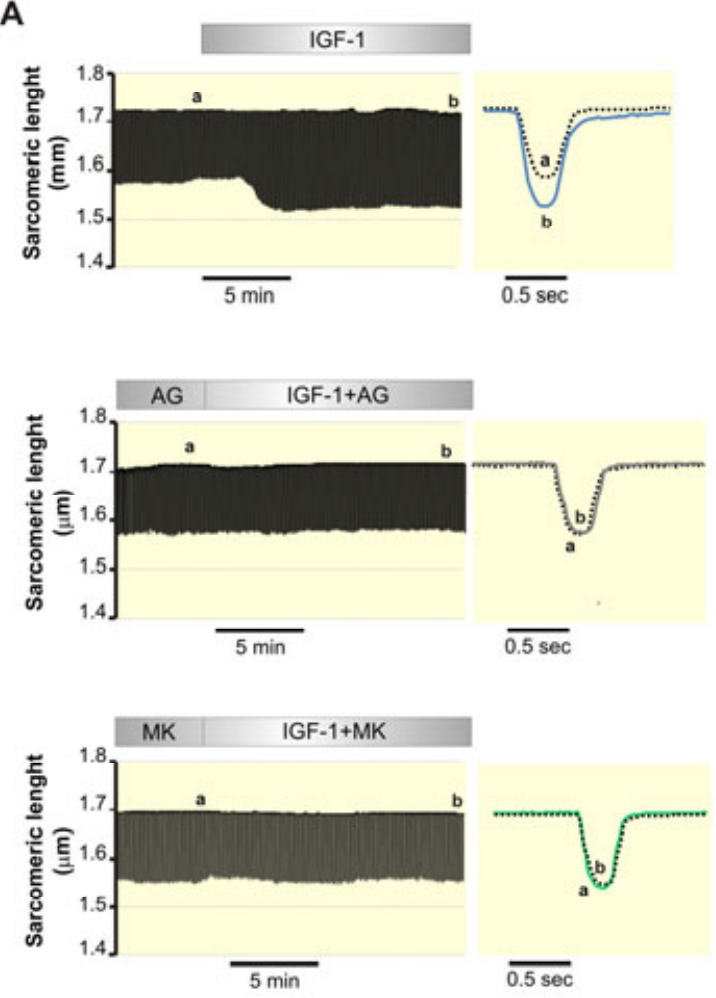

B
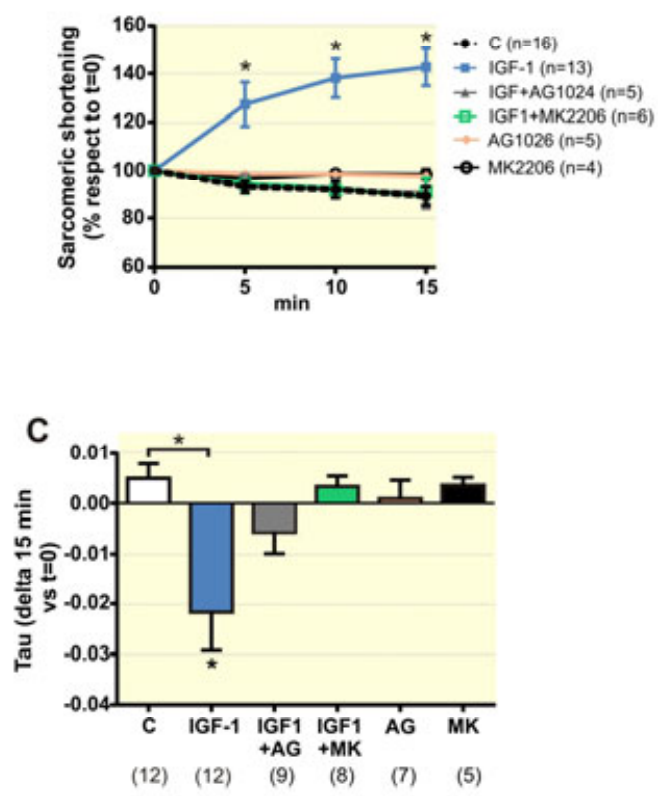

This article is protected by copyright. All rights reserved. 
A

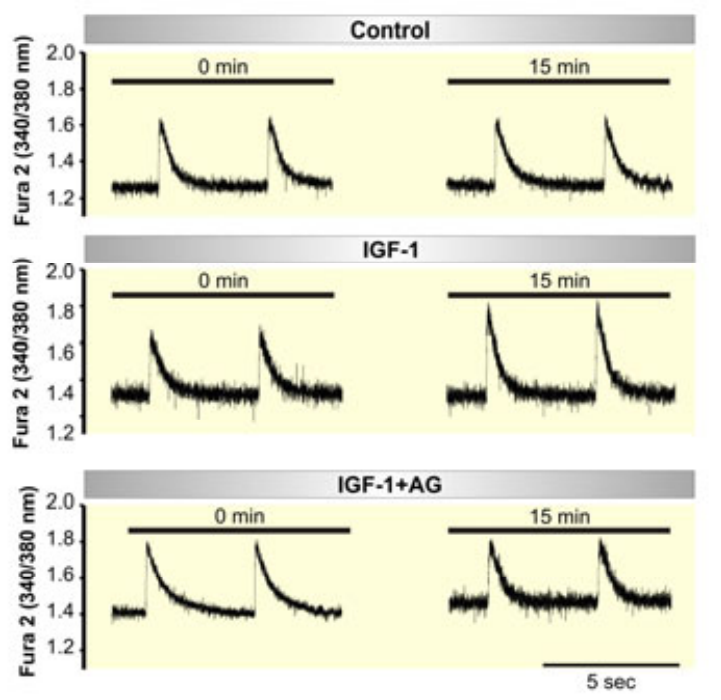

D
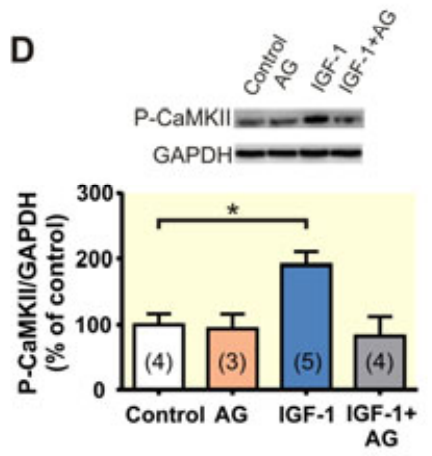

B
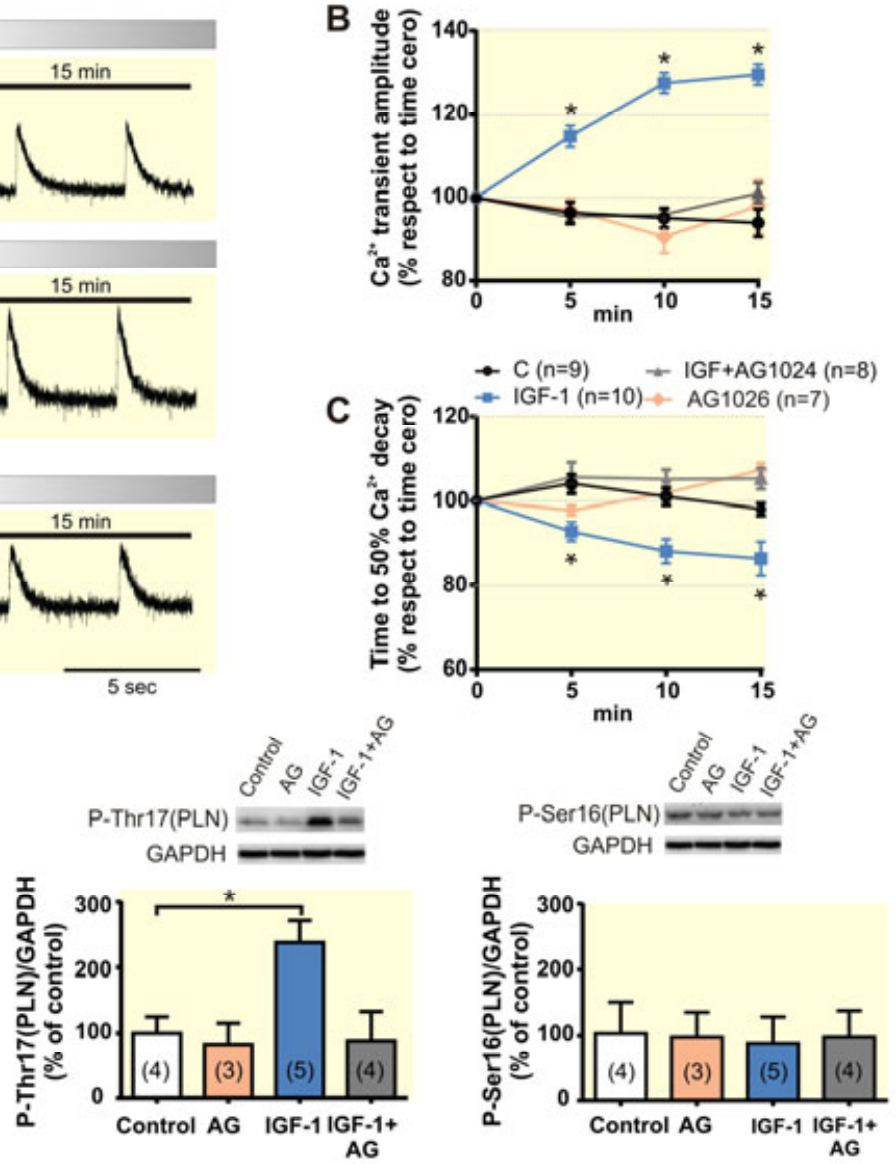

This article is protected by copyright. All rights reserved. 

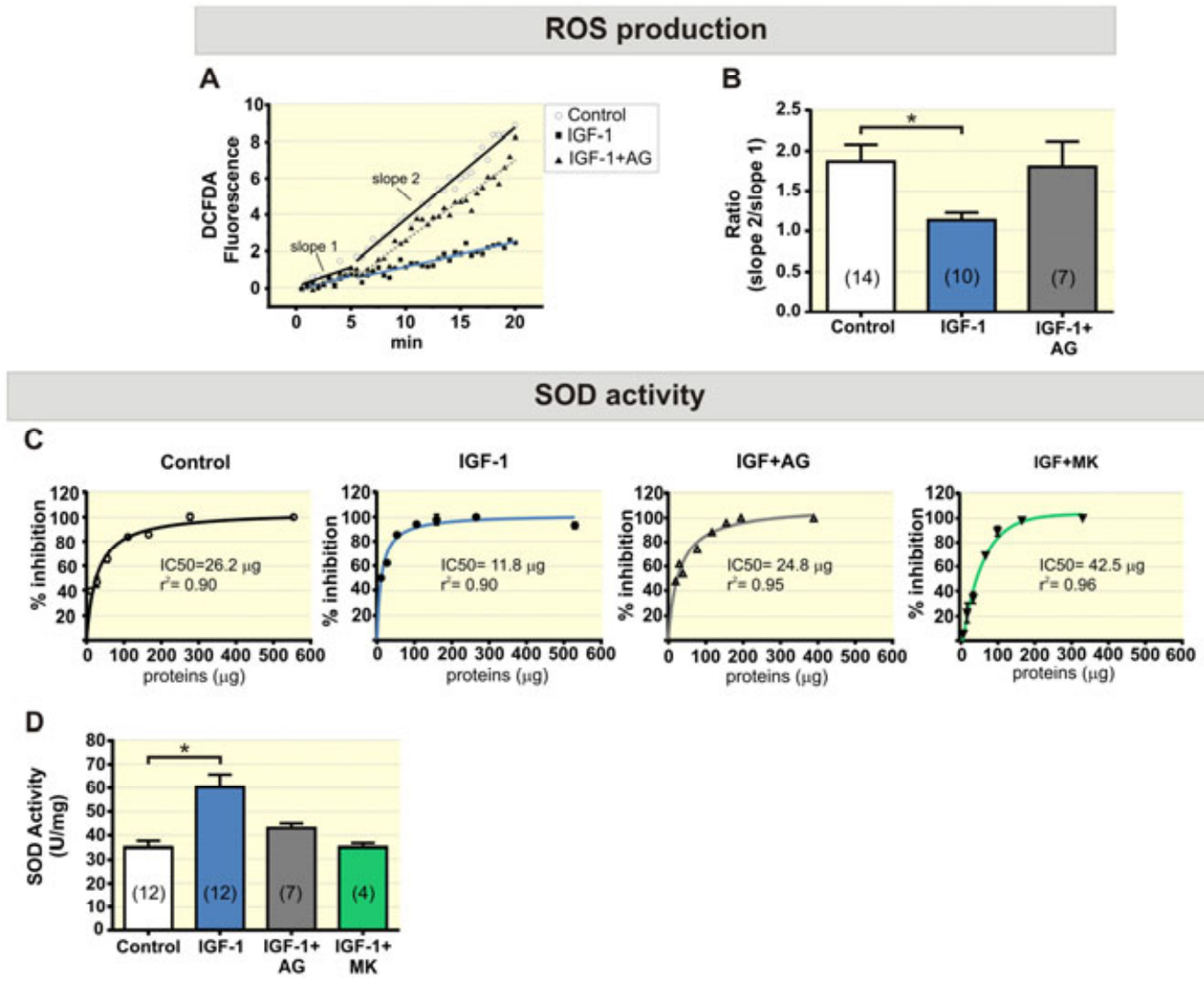

This article is protected by copyright. All rights reserved. 


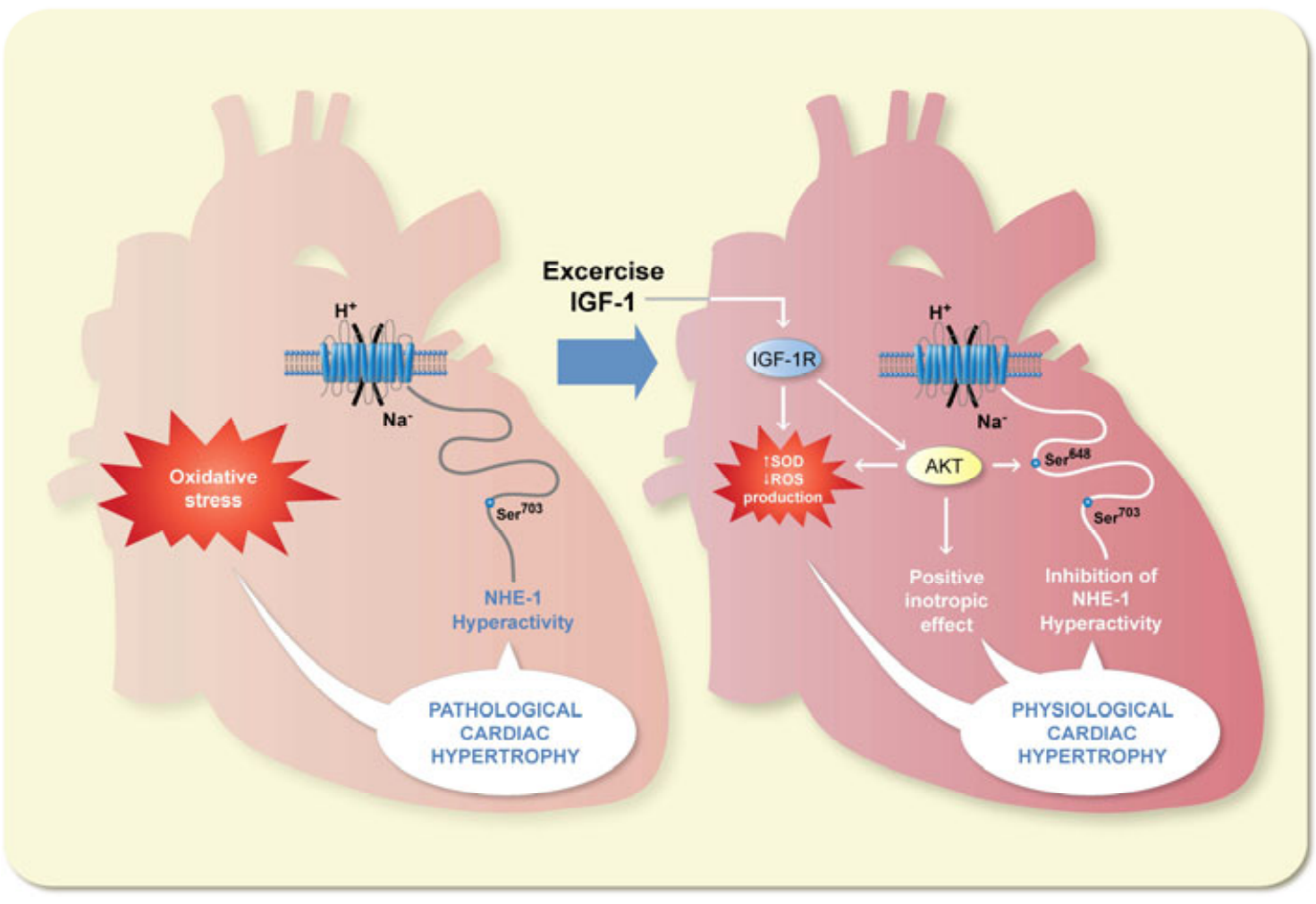

This article is protected by copyright. All rights reserved. 